\title{
The cameroon mobile phone sms (CAMPS) trial: a protocol for a randomized controlled trial of mobile phone text messaging versus usual care for improving adherence to highly active anti-retroviral therapy
}

${\text { Lawrence Mbuagbaw }{ }^{1 *} \text {, Lahana Thabane }}^{2,3}$, Pierre Ongolo-Zogo ${ }^{1}$, Richard T Lester ${ }^{4,5}$, Edward Mills ${ }^{6}$, Jimmy Volmink ${ }^{7}$, David Yondo ${ }^{1}$, Marie José Essi ${ }^{1}$, Renée-Cecile Bonono-Momnougui ${ }^{1}$, Robert Mba', Jean Serge Ndongo ${ }^{1}$, Francois C Nkoa', Henri Atangana Ondoa ${ }^{1}$

\begin{abstract}
Background: This trial aims at testing the efficacy of weekly reminder and motivational text messages, compared to usual care in improving adherence to Highly Active Antiretroviral Treatment in patients attending a clinic in Yaoundé, Cameroon.

Methods and Design: This is a single-centered randomized controlled single-blinded trial. A central computer generated randomization list will be generated using random block sizes. Allocation will be determined by sequentially numbered sealed opaque envelopes. 198 participants will either receive the mobile phone text message or usual care. Our hypothesis is that weekly motivational text messages can improve adherence to Highly Active Antiretroviral Treatment and other clinical outcomes in the control group by acting as a reminder, a cue to action and opening communication channels. Data will be collected at baseline, three months and six months. A blinded program secretary will send out text messages and record delivery.

Our primary outcomes are adherence measured by the visual analogue scale, self report, and pharmacy refill data. Our secondary outcomes are clinical: weight, body mass index, opportunistic infections, all cause mortality and retention; biological: Cluster Designation 4 count and viral load; and quality of life. Analysis will be by intentionto-treat. Covariates and subgroups will be taken into account.
\end{abstract}

Discussion: This trial investigates the potential of SMS motivational reminders to improve adherence to Highly Active Antiretroviral Treatment in Cameroon. The intervention targets non-adherence due to forgetfulness and other forms of non-adherence.

Trial Registration: Pan-African Clinical Trials Registry PACTR201011000261458 http://clinicaltrials.gov/NCT01247181

\footnotetext{
* Correspondence: mbuagbawl@yahoo.com

${ }^{1}$ Centre for the Development of Best Practices in Health(CDBPH), Yaoundé Central Hospital, Avenue Henri Dunant, Messa, PO Box 87, Yaoundé,

Cameroon

Full list of author information is available at the end of the article
} 


\section{Background}

Mobile text messages using the short message service (SMS) are a cheap and non-invasive means of communication that can be used to convey health related messages to owners of mobile phones. There is contradictory evidence concerning the role of mobile phones in ameliorating health outcomes, especially in less developed countries where private ownership and use of mobile phones is not as widespread as in other more developed countries [1]. Currently, Africa has the greatest uptake of mobile phone technology [2].

The advent of Highly Active Antiretroviral Therapy (HAART) has markedly reduced morbidity and mortality associated with the Human Immune deficiency Virus (HIV) [3]. Much effort has been put into the scaling up of access to HAART [4]. The efficacy of HAART depends largely on compliance to treatment regimens. Poor adherence is associated with poor virological and immunological response. It is also responsible for the development of resistant strains [5]. Very high levels (> 95\%) of adherence are necessary for sustained clinical success [6].

Our search for papers on the use of SMS technology to improve adherence to HAART revealed two protocols for trials in Kenya (WelTel Kenya 1) [7] and India (HIVIND) [8]. The WelTel Kenya 1 trial reported significant improvements in adherence and viral load [9].

The World Health Organization (WHO) has prioritized the use of new technologies to assist health delivery in resource-limited settings [10]. The SMS is already used for business transactions, personal communication, advertising and betting. There is a potential for new benefits to be discovered in the use of mobile phone technology in health interventions for resource- limited countries [11]. In South Africa, the SMS has been demonstrated to improve HIV health care service delivery by ameliorating communication between health workers and patients, and also as an appointment reminder [12].

Additionally, a Cochrane systematic review found that mobile phone calls and reminders can improve adherence to tuberculosis care [13]. Another study investigating the use of mobile phone technology to improve adherence to HAART discouraged the use of phone calls as they are time and labor intensive [14]. A third study, investigating the use of text messaging to improve adherence to primary care found that it was more cost effective than phone calls[15]. The cost of phone calls may also be a hindrance in developing countries. The Indian trial [8] is testing automated phone calls coupled to picture messages. This may reduce the time and labor costs involved. Another study reported high satisfaction with two way text messaging [16]. These findings suggest that the more feasible application of the mobile phone in health would be the SMS.
The goal of this trial is to assess whether sending weekly motivational text messages via a mobile phone versus no text messaging will improve adherence to HAART and clinical outcomes among HIV positive patients over a 6 month period. We hypothesize that sending one weekly motivational reminder will produce a change in behavior to enhance drug or treatment adherence and hence clinical outcomes. SMS-delivered interventions are capable of producing a positive behavior change [17].

Participants will be selected from the Accredited Treatment Centre (ACT) of the Yaoundé Central Hospital $(\mathrm{YCH})$.

\section{Methods and Design}

The trial is registered with the Pan-African Clinical Trials Registry http://pactr.org as PACTR201011000261458 and with clinical trials.gov as NCT01247181.

\section{Funding}

Partial funding for this study was obtained from an international postdoctoral research fellowship awarded by the Canadian Institutes of Health Research (CIHR) HIV Clinical Trials Network (CTN) to the principal investigator.

\section{Study Design}

Using a 1:1 allocation ratio, patients at the Yaoundé Central Hospital ( $\mathrm{YCH})$ Accredited Treatment Centre (ATC) will be randomized to either receive a text message reminder to take their medication or not (Figure 1: Consolidated Standards of Reporting Trials-CONSORT diagram of study design). Both groups of patients will benefit from the usual care provided in this centre which includes adherence counseling and rarely, home visits.

\section{Randomization}

This is a parallel group design evaluating the effects of adding weekly SMS text messages using mobile phones to usual care (intervention) versus usual care alone (control) among HIV positive patients on HAART. Eligible and consenting patients will be randomized to intervention and control arms using 1:1 allocation ratio by opaque sealed envelope method. A computer generated randomization list will be generated using random block sizes of 2, 4 and 6, by the Father Sean O'Sullivan Research Centre Biostatistics Unit at St Joseph's Healthcare/McMaster University. The allocation codes will then be put in sequentially numbered opaque sealed envelopes and administered by the trained Research Staff at YCH ATC centre. Trained interviewers - blinded to group allocation - will collect data using a pretested 


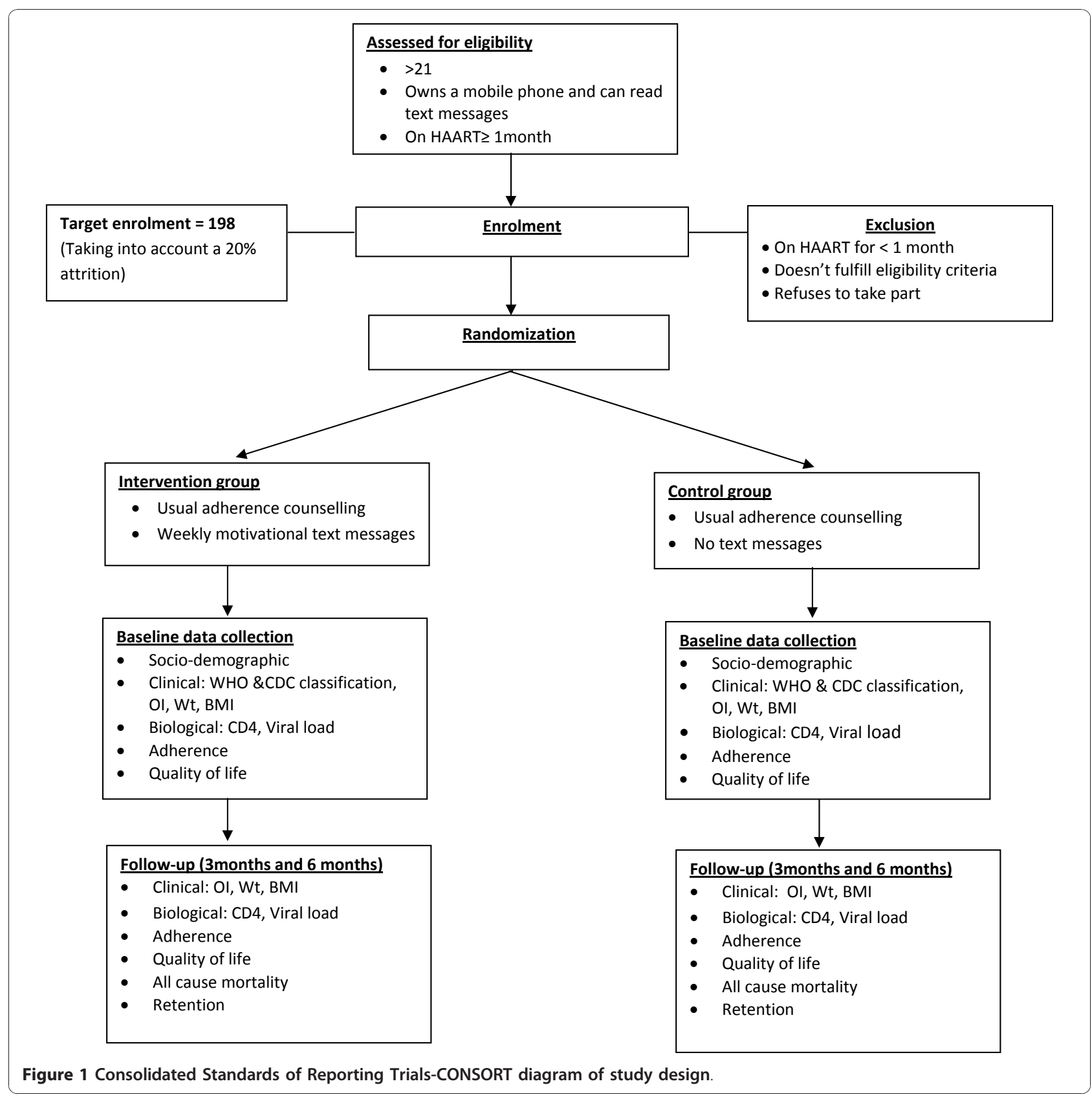

data collection form containing socio-demographic data, clinical information and adherence rates at baseline, 3 and 6 months. The data analyst will also be blinded to group allocation.

\section{Trial Setting}

Cameroon is a sub-Saharan central African country, made of ten provinces and a population of 18 million inhabitants [18]. The Centre and capital province has a population of about 3.2 million inhabitants. The adult prevalence rate of HIV in the country is 5.1\% [19]. Subjects will be recruited from the $\mathrm{YCH}$ ACT. This is an urban centre in the heart of the capital city, Yaoundé. The adult prevalence of HIV in the Centre province is $4.7 \%$. The $\mathrm{YCH}$ is a tertiary level general teaching hospital with a capacity of 381 beds. It employs nearly 800 staff including 95 doctors and 270 nurses [20]. The ACT has a very high recruitment rate of approximately 40 new cases per week and caters for 6500 regular clients. It is the largest HIV clinic in the country and offers enormous potential for recruitment.

\section{Participants: inclusion/exclusion criteria}

We will include subjects who are aged above 21 years, own a mobile phone and can read text messages, and 
who have been on HAART for at least a month. Informed consent is a prerequisite for participating in the study, and will be provided orally and in writing.

We will exclude participants who have been on HAART for less than a month, are aged less than 21 years. Participants who have used HAART for at least one month are chosen so that we can calculate a baseline figure for adherence.

\section{Intervention}

We will send a short text message to the participants in the intervention group in both French and English. The content of the message will be motivating and will act both as a reminder and a cue to action (Figure 2: Example of a text message). The message will also contain a phone number they can call back if they need help. The content will be varied so as to retain participants' attention throughout the period of the study and to explore the various aspects of behavior change. The program secretary will have a list of phone numbers to which he/ she will send the messages every week and will use the 'delivery report' function to ensure that the messages have been delivered. One message will be sent per week in the morning of a chosen day. The average cost for text messages on any networks is 50 CFA Frs. CFA

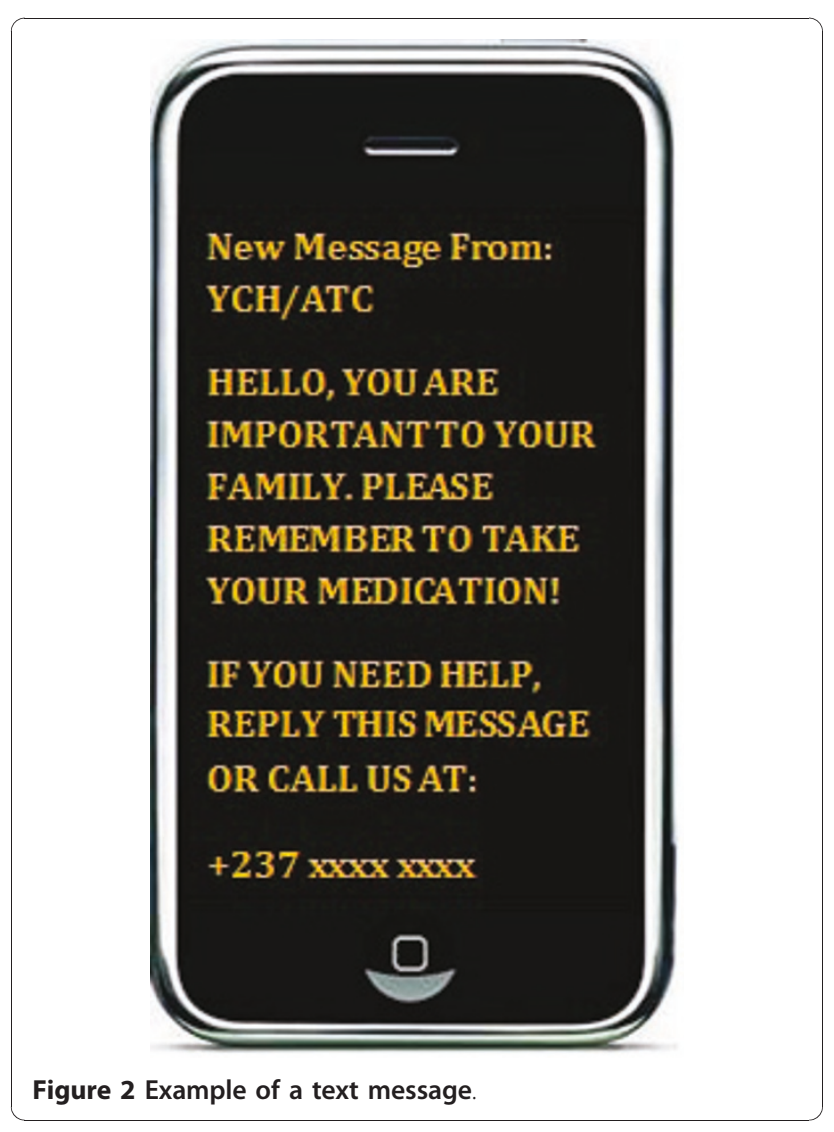

( $\approx 0.1$ USD). Text messaging will be provided as an addon to usual care which includes regular HAART counseling and occasional home visits.

\section{Control}

In the control arm, patients will receive the usual care provided to all patients of the ATC which includes regular HAART counseling and occasional home visits. They will be given a number to call if they have questions. They will not receive any text messages, but they will be interviewed at baseline, 3 months and 6 months.

\section{Study Objectives \\ Primary Objective}

The primary objective of this trial is to investigate the effect of adding the SMS to usual care versus usual care alone in improving and maintaining adherence to HAART in HIV positive patients on HAART at 3 and 6 months. There are several methods used to evaluate or measure adherence to medications, each with advantages and disadvantages $[21,22]$. Thus, there is no gold standard in measuring medication adherence $[21,23]$. The common approach is to use multiple methods to compare or assess the robustness of the estimates of adherence. For this study, we will use three commonly used measures of adherence - namely Visual Analogue Scale (VAS), Pharmacy Refill Data (PRD), and Self Report (SR). We will use VAS as the primary method of measuring adherence. The VAS is highly correlated with more objective methods like using Microelectronic Monitoring System (MEMS) caps [24]. VAS method has also been widely used in several RCTs evaluating different interventions including mobile text messaging to enhance adherence to HAART $[8,21]$. PRD and SR will be used to fill any missing data on VAS.

\section{Secondary objectives}

The secondary objectives include comparing clinical outcomes such as weight, body mass index (BMI), opportunistic infections (OI), Cluster Designation (CD) 4 count, viral load and quality of life between the groups. These comparisons will be performed at 3 and 6 months.

\section{Outcome measures}

Our primary outcome will be adherence rates, measured using VAS. SR and PRD will also be used to supplement VAS (Table 1).

Our Secondary endpoints will be;

- Clinical: Weight, BMI, opportunistic infections

- Biological: CD4 count, viral load

- Quality of life (QOL): Measured with the SF-12 QOL assessment form [25].

- All cause mortality

- Retention 
Table 1 Overview of outcome measures

\begin{tabular}{|c|c|c|c|c|}
\hline Outcome measures & Scale & Type & Measure & Analysis method \\
\hline \multicolumn{5}{|l|}{ Primary } \\
\hline \multicolumn{5}{|l|}{ Adherence at 6 months* } \\
\hline VAS & Ordinal & Binary & VAS percentage $>95 \%$ & Chi-squared test \\
\hline Self report & Ordinal & Binary & $\%$ adherence in last month $>95 \%$ & Chi-squared test \\
\hline PRD & Ordinal & Binary & $\%$ of complete refills $>95 \%$ & Chi-squared test \\
\hline \multicolumn{5}{|l|}{ Secondary } \\
\hline Weight & Ratio & Continuous & Change in weight & T-test \\
\hline $\mathrm{BMI}$ & Ratio & Continuous & Change in BMI & T-test \\
\hline $\mathrm{Ol}$ & Nominal & Binary & Occurrence of new Ol & Chi-squared test \\
\hline Mortality & Nominal & Binary & All deaths & Chi-squared test \\
\hline Retention & Nominal & Binary & Number retained in care & Chi-squared test \\
\hline CD4 count & Ratio & Continuous & Change in CD4 count & T-test \\
\hline Viral load & Ratio & Continuous & Change in viral load & T-test \\
\hline Satisfaction with care & Ordinal & Categorical & Change in satisfaction scores & T-test \\
\hline QoL & Ordinal & Categorical & Change in QoL scores & T-test \\
\hline
\end{tabular}

VAS: visual analogue scale, PRD: pharmacy refill data, BMI, body mass index, Ol: opportunistic infection, CD4: Cluster Designation 4, QoL: Quality of Life. *All three measures of adherence will also be analyzed as continuous outcomes using T-test.

\section{Duration}

The trial will run for six months, with outcome assessment at baseline, 3 months and 6 months.

\section{Sample size}

The sample size calculation is based on the test of the null hypothesis that the rates of adherence to HAART in the two groups (intervention and control) are equal. The primary measure of effect is the rate of adherence to ART treatment as measured by using the VAS over 6 months. The criterion for significance (alpha) has been set at 0.05. The test is 2-tailed, which means that an effect in either direction will be interpreted. The sample size was calculated using the WINPEPI (PEPI- for-windows) version 9.5 software [26]. With the proposed sample size of 82 in each of the two groups (i.e. assuming a $1: 1$ allocation ratio), the study will have power of $80 \%$ to yield a statistically significant result using a chi-squared test (assuming an intention-to-treat principle for the analysis) of the relative risk at alpha $=0.05$. This computation assumes an adherence rate of $80 \%$ (for the intervention group) versus $60 \%$ (for the control group) at 6 months. These estimates are reflective of estimates from similar studies investigating SMS effect on drug adherences [27] and were modified to account for the type of intervention and patients for this study. We adjusted the sample size for a potential attrition rate of $20 \%$ (due to drop-outs) based on attrition rates to care in this centre. Therefore, the required sample size is 198 patients (99 per group). At the YCH ATC, on average, there are about 120 patients put on HAART per month. We estimate that about $90 \%$ will have mobile phones and would be eligible to participate in the study. Of these, it is expected that approximately $75 \%$ would be willing to participate in the study and will provide consent to participate in the trial. The expected period for recruitment will be one month to obtain 198 patients needed for the trial. It is feasible to recruit 198 patients in one month because we will also recruit from the large pool of old patients. Our study is designed to detect a $20 \%$ increase in adherence.

\section{Analysis plan}

The analysis and reporting of the results with follow the CONSORT guidelines [28]. The statistician/data analyst will be blinded to the study group. The process of patient selection and flow throughout the study will be summarized using a flow-diagram. The analysis results of patient demographics and baseline outcome variables (both primary and secondary) will be summarized using descriptive summary measures: expressed as mean (standard deviation) or median (minimum-maximum) for continuous variables and number (percent) for categorical variables. We will adopt an intention-to-treat principle to analyze all outcomes, meaning that data from participants will be analyzed according to the group to which they were randomized even if they do not receive the allocated intervention. We will also use multiple-imputation [29] to handle missing data. We will use the T-test for comparing groups on continuous outcomes and the chi-squared test for binary outcomes. All statistical tests will be performed using two-sided tests at the 0.05 level of significance. The Bonferroni method will be used to adjust the level of significance for testing for secondary 
outcomes to keep the overall level at alpha $=0.05$. For all group comparisons, the results will be expressed as effect (or risk ratio for binary outcomes), corresponding twosided $95 \%$ confidence intervals and associated p-values. $\mathrm{P}$-values will be reported to three decimal places with values less than 0.001 reported as $<0.001$. Adjusted analyses using the following baseline covariates (age, gender, education, duration on HAART, HIV staging, nutritional status (BMI) and the presence or not of an opportunistic infection (OI)) will be performed using regression techniques to investigate the residual impact of key baseline characteristics on the outcomes. Goodness-of-fit will be assessed by examining the residuals for model assumptions and chi-squared test of goodness-of-fit. All analyses will be performed using SPSS (Statistical Package for the Social Sciences) version 16.0 for Windows.

Adherence will be measured both as a continuous outcome (change in adherence) and as a binary outcome i.e. adherent (95\% of pills taken) or non adherent $(<95 \%$ of pills taken). In literature, adherence data can be handled in a number of ways. The measures can be reported as the number of doses respected or can be combined into a composite score [21]. Even though combined measures are more correlated to clinical response, they are not very practical [22]. The data from the various adherence measures will not be merged. We will report the effects of the intervention on all the measures of adherence used, and compare them for discrepancies.

\section{Additional studies}

- Adherence rates: We will use this opportunity to calculate the rates of adherence to HAART in the YCH ATC. The context of adherence to HAART in Cameroon has changed greatly over time and the rates reported in literature were subject to cost (which has been dropping over time), availability, accessibility, study design, technique used to measure adherence and study setting. These rates vary from $10 \%$ to $97 \%$ [30-32]. This study will provide a more accurate and current estimate of adherence in Cameroon.

- Safety: Data will be collected on issues that may arise from the use of text messages to improve adherence.

- Health worker experiences: Self-administered questionnaires will be used to assess health worker perceptions of the intervention in terms of long term use, additional workload and benefits to care. The applicability of such an intervention will depend largely on its acceptability by health workers.

\section{Ethical Considerations}

The trial will be conducted in compliance with the local protocol and applicable regulatory requirements in
Cameroon. The study has been approved by Cameroon National Ethics Committee. Any deviations from the protocol will be reported and explained. The study will be conducted in accordance with the Helsinki declaration [33] and other established clinical practice guidelines for research on human subjects. Research personnel will approach all potentially eligible patients who fulfill eligibility criteria for consent. All patients must sign a consent form to participate in the trial.

\section{Discussion}

The potential of mobile phone technology to improve health outcomes is a domain worth exploring, especially in this era of increased uptake and dependence on mobile phones. Studies investigating the use of text messages to improve adherence have yielded varied results $[12,16,34]$.

Improving adherence to HAART can play a key role in reducing morbidity and mortality due to HIV, the occurrence of drug resistant strains and the waste of medication in health systems that are already seriously challenged by the advent of HIV. Findings generated from this trial may be generalized to other chronic illnesses.

A major ethical consideration is the harm that may arise due to accidental disclosure of status. This eventuality will be properly explained to the participant, even though our message will neither disclose status nor medication. In a study evaluating the use of mobile phones to improve attendance to an HIV clinic in Uganda, privacy and confidentiality were not a problem [35]. Loss of privacy was not identified as a deterrent in the HIVIND trial $[8,36]$. We are aware that most mobile operators in Cameroon deliver mass text messages to their clients for business, advertising, information and sometimes health. Our intervention will be an addition to an already existing system. Another concern is how to manage text messaging on a larger scale, knowing fully well that it cannot be left in the hands of mobile operators, who will then be privy to the phone numbers of potentially identifiable individuals. In this trial, the operators will not be used because a framework for cooperation that will not jeopardize confidentiality and anonymity hasn't been established. A single phone with prepaid airtime will be used to deliver the text messages.

This intervention centers on the Health Belief Model of behavior change [37]. We will collect data on modifying factors (socio-demographic and disease related), perceived barriers to adherence and test the efficacy of the SMS reminder as a cue to action. Putting all these in context will provide a better picture of who doesn't adheres to HAART, why they don't adhere and if text messages can help. Applying the SMS not only as a reminder, but as a cue to action permits it use to resolve 
intentional and non-intentional non-adherence. In addition, we will open channels for communication between appointments. Galovotti et al succinctly describe the key components of an intervention aimed at bringing about a change in behavior. The use of role models, affective impact, and links to socio-cultural narratives, personalization and knowledge of impediments and facilitators are important features of successful behavioral interventions [38].

Finally, this trial may contribute to the growing body of evidence on the use of mobile phone technology to improve health outcomes in low resource settings.

\section{Abbreviations \\ ATC: Accredited Treatment Centre; BMI: Body Mass Index; CD: Cluster Designation; CIHR: Canadian Institutes for Health Research; CONSORT: Consolidated Standards for Reporting Trials; CTN: Canadian HIV Trials Network; HAART: Highly Active Anti-Retroviral Therapy; HIV: Human Immune Deficiency Virus; MEMS: Micro-Electronic Monitoring System; Ol: \\ Opportunistic Infections; PRD: Pharmacy Refill Data; QOL: Quality of Life; SMS: Short Message Service; SPSS: Statistical Package for Social Sciences; SR: Self Report; VAS: Visual Analogue Scale; WHO: World Health Organization; YCH: Yaoundé Central Hospital.}

\section{Acknowledgements}

This study is support in part by Canadian Institutes of Health Research (CIHR) HIV Clinical Trials Network (CTN) in the form of international postdoctoral research fellowship award to the first author. Dr Thabane is a clinical trials mentor for CIHR under the RCT Mentorship Programme.

\section{Author details \\ 'Centre for the Development of Best Practices in Health(CDBPH), Yaoundé Central Hospital, Avenue Henri Dunant, Messa, PO Box 87, Yaoundé, Cameroon. ${ }^{2}$ Department of Clinical Epidemiology and Biostatistics, McMaster University, Hamilton, ON, Canada. ${ }^{3}$ Biostatistics Unit, Father Sean O'Sullivan Research Centre, St Joseph's Healthcare, Hamilton, ON, Canada. ${ }^{4}$ Department of Medicine, Division of Infectious Diseases University of British Columbia, Vancouver, BC, Canada. ${ }^{5}$ British Columbia Centre for Disease Control, Vancouver, BC, Canada. ${ }^{6}$ Faculty of Health Sciences, University of Ottawa, Ottawa, ON, Canada. 'Faculty of Health Sciences Stellenbosch University, Capetown, South Africa.}

\section{Authors' contributions}

LM conceived of the study. LT, POZ, RTL, EM, JV helped to draft the manuscript. DY, MJE, RCBM, RM, JSN, FN and HAO participated in its design and helped draft the manuscript. All authors read and approved the final manuscript.

\section{Competing interests}

The authors declare that they have no competing interests.

Received: 26 November 2010 Accepted: 7 January 2011

Published: 7 January 2011

\section{References}

1. Kaplan AW: Can the ubiquitous power of mobile phones be used to improve health outcomes in developing countries? Global Health 2006, 2:9.

2. Lester RT, Gelmon L, Plummer FA: Cellphones: tightening the communication gap in resource-limited antiretroviral programmes? AIDS 2006, 20:2242-2244.

3. Mocroft A, Vella S, Benfield TL, Chiesi A, Miller V, Gargalianos P, d'Arminio Monforte A, Yust I, Bruun JN, Phillips AN, Lundgren JD: Changing patterns of mortality across Europe in patients infected with HIV-1. EuroSIDA Study Group Lancet 1998, 28;352:1725-30.
4. UNAIDS: Report on global AIDS epidemic. 2009 [http://www.unaids.org/ en/media/unaids/contentassets/dataimport/pub/report/2009/ jc1700_epi_update_2009_en.pdf], (Accessed November 15, 2009).

5. Gill CJ, Davidson HH, Jonathon LS, Donald MT, Lora LS: No Complacency about adherence to antiretroviral therapy in sub-saharan Africa. AIDS 2005, 19:1243-1249.

6. Paterson DL, Swindells S, Mohr J: Adherence to protease inhibitor therapy and outcomes in patients with HIV infection. Ann Intern Med 2000, 133:21-30.

7. Lester R, Mills E, Kariri A, Ritvo P, Chung M, Jack W, Habyarimana J, Karanja S, Barasa S, Nguti R, Estambale B, Ngugi E, Ball TB, Thabane L, Kimani J, Gelmon L, Ackers M, Plummer FA: The HAART cell phone adherence trial (WelTel Kenya1): a randomized controlled trial protocol. Trials 2009, 10:87.

8. De Costa A, Shet A, Kumarasamy N, Ashorn P, Eriksson B, Bogg L, Diwan V, team tHs: Design of a randomized trial to evaluate the influence of mobile phone reminders on adherence to first line antiretroviral treatment in South India - the HIVIND study protocol. BMC Med Res Methodol 2010, 10:25.

9. Lester RT, Ritvo P, Mills EJ, Kariri A, Karanja S, Chung MH, Jack W, Habyarimana J, Sadatsafavi M, Najafzadeh M, Marra CA, Estambale B, Ngugi E, Ball TB, Thabane L, Gelmon L, Kimani J, Ackers M, Plummer FA: Effects of a mobile phone short message service on antiretroviral treatment adherence in Kenya (WelTel Kenya 1): a randomized trial. Lancet 2010.

10. World Health Organization: UNAIDS: Resource needs for an expanded response to AIDS in low and middle-income countries. [http://data. unaids.org/pub/Report/2005/jc1255_resource_needs_en.pdf], (Accessed November 20, 2010).

11. Lester R, Karanja S: Mobile phones: exceptional tools for HIV/AIDS, health, and crisis management. Lancet Infect Dis 2008, 8:738-739.

12. Mukund Bahadur KC, Murrayb PJ: Cell Phone Short Messaging Service (SMS) for HIV/AIDS in South Africa: A literature review. Stud Health Technol Inform 2010, 160:530-534.

13. Liu Q, Abba K, Alejandria MM, Balanag VM, Berba RP, Lansang MAD: Reminder systems and late patient tracers in the diagnosis and management of tuberculosis. Cochrane Database of Systematic Reviews 2008, 4: CD006594, Evidence Update published in June 2010.

14. Wiener L, Riekert K, Ryder C, Wood LV: Assessing medication adherence in adolescents with HIV when electronic monitoring is not feasible. AIDS Patient Care STDS 2004, 18:527-538.

15. Leong KC, Chen WS, Leong KW, Mastura I, Mimi O, Sheikh MA, Zailinawati $\mathrm{AH}, \mathrm{Ng} \mathrm{CJ}$, Phua $\mathrm{KL}$, Teng $\mathrm{CL}$ : The use of text messaging to improve attendance in primary care: a randomized controlled trial. Fam Pract 2006, 23:699-705.

16. Dunbar PJ, Madigan D, Grohskopf LA, Revere D, Woodward J, Minstrell J, Frick PA, Simoni JM, Hooton TM: A two-way messaging system to enhance antiretroviral adherence. J Am Med Inform Assoc 2003, 10:11-15.

17. Brianna S Fjeldsoe, Alison L Marshall, Yvette D Miller: Behavior Change Interventions Delivered by MobileTelephone Short-Message Service. Am J Prev Med 2009, 36(2):165-173.

18. World Health Organization: Cameroon. 2010 [http://www.who.int/countries/ cmr/en/], (Accessed November 8, 2010).

19. UNICEF: Cameroon statistics. 2010 [http://www.unicef.org/infobycountry/ cameroon_statistics.html], (Accessed November 8, 2010).

20. World Health Organization: Yaoundé, Cameroon-HUG, Switzerland. 2010 [http://www.who.int/patientsafety/implementation/apps/first_wave/ cameroon_hug/en/, (Accessed November 13, 2010).

21. Simoni J, Kurth A, Pearson C, Pantalone D, Merrill J, Frick P: Self-Report Measures of Antiretroviral Therapy Adherence: A Review with Recommendations for HIV Research and Clinical Management. AIDS Behav 10:227-245.

22. Liu H, Golin CE, Miller LG, Hays RD, Beck CK, Sanandaji S, Christian J, Maldonado T, Duran D, Kaplan AH, Wenger NS: A Comparison Study of Multiple Measures of Adherence to HIV Protease Inhibitors. Ann Intern Med 2001, 134:968-977.

23. Martin S, Elliott-DeSorbo DK, Calabrese S, Wolters PL, Roby G, Brennan T, Wood LV: A comparison of adherence assessment methods utilized in the United States: perspectives of researchers, HIV-infected children, and their caregivers. AIDS Patient Care STDS 2009, 23:593-601. 
24. Arnsten JH, Demas PA, Farzadegan H, Grant RW, Gourevitch MN, Chang CJ, Buono D, Eckholdt H, Howard AA, Schoenbaum EE: Antiretroviral therapy adherence and viral suppression in HIV-infected drug users: comparisonof self-report and electronic monitoring. Clin Infect Dis 2001, 33:1417-23.

25. SF Tools: The SF-12: An even shorter Health Survey. [http://www.sf-36. org/tools/sf12.shtml], (Accessed November 9, 2010).

26. Abramson JH: WINPEPI (PEPI-for-Windows): computer programs for epidemiologists. Epidemiologic Perspectives \& Innovations 2004, 1:6.

27. Strandbygaard $U$, Thomsen SF, Backer V: A daily SMS reminder increases adherence to asthma treatment: a three-month follow-up study. Respir Med 2010, 104:166-171.

28. Schulz KF, Altman DG, Moher D: CONSORT 2010 Statement: Updated guidelines for reporting parallel group randomised trials. J Clin Epidemiol 2010, 63:834-840.

29. Little RJA, Rubin DB: Statistical analysis with missing data. New York: John Wiley \& Sons; 1987.

30. Mosoko JJ, Akam W, Weidle PJ, Brooks JT, Aweh AJ, Ebad M, Kinge TN, Vitek C, Raghunathan PL: Survival and adherence to antiretroviral therapy in an era of decreasing drug cost in Limbe, Cameroon. Proceedings of the 14th Conference on Retroviruses and Opportunistic Infections (CROI): 25-28 February 2007; Los Angeles, California. Abstract 536 [http://www. retroconference.org/2007/, (Accessed 28 July 2010).

31. Charles Kouanfack, Christian Laurent, Gilles Peytavin, Yvette Mawamba Nkene, Claudine Essomba, Laura Ciaf, Maguy Ngolle, Eric Delaporte, Alexandra Calmy, Eitel Mpoudi-Ngole', SinataKoulla Shiro, for the French National Agency for Research on AIDS, 1274 Study Group: Adherence to Antiretroviral Therapy Assessed by Drug Level Monitoring and Self-Report in Cameroon. J Acquir Immune Def Syndr 2008, 48:216-219.

32. Boyer S, Marcellin F, Ongolo-Zogo P, Abega SC, Nantchouang R, Spire B, Moatti JP: Financial barriers to HIV treatment in Yaoundé, Cameroon: first results of a national cross-sectional survey. Bull World Health Organ 2009, 87:279-287.

33. World Medical Organization: Declaration of Helsinki. BMJ 1996, 313:1448-1449.

34. Reynolds NR, Testa MA, Su M, Chesney MA, Neidig JL, Frank I, Smith S, Ickovics J, Robbins GK: Telephone support to improve antiretroviral medication adherence: a multisite, randomized controlled trial. J Acquir Immune Defic Syndr 2008, 47:62-68.

35. Kunutsor S, Walley J, Katabira E, Muchuro S, Balidawa H, Namagala E, Ikoona E: Using mobile phones to improve clinic attendance amongst antiretroviral treatment cohort in rural Uganda: A cross-sectional and prospective study. AlDS Behav 2010.

36. Shet A, Arumugam K, Rodrigues R, Rajagopalan N, Shubha K, Raj T, D'Souza G, De Costa A: Designing a mobile phone-based intervention to promote adherence to antiretroviral therapy in South India. AIDS Behav 2010, 14:716-720.

37. Maimen LA, Becker MH: The health belief model: origins and correlates in psychological theory. Health Educ Monogr 1974, 2:336-53.

38. Galavotti C, Pappas-DeLuca KA, Lansky A: Modeling and Reinforcement to Combat HIV: The MARCH Approach to Behavior Change. Am J Public Health 2001, 91:1602-1607.

doi:10.1186/1745-6215-12-5

Cite this article as: Mbuagbaw et al:: The cameroon mobile phone sms (CAMPS) trial: a protocol for a randomized controlled trial of mobile phone text messaging versus usual care for improving adherence to highly active anti-retroviral therapy. Trials 2011 12:5.

\section{Submit your next manuscript to BioMed Central and take full advantage of:}

- Convenient online submission

- Thorough peer review

- No space constraints or color figure charges

- Immediate publication on acceptance

- Inclusion in PubMed, CAS, Scopus and Google Scholar

- Research which is freely available for redistribution

Submit your manuscript at www.biomedcentral.com/submit
Biomed Central 